\title{
Correlação entre o hemocitômetro e outras técnicas de rotina para a contagem do número de plaquetas em cães atendidos no Hospital Veterinário da Universidade Estadual de Londrina (H.V.-UEL)
}

\author{
Correlation between the haemocytometer and others routine \\ techniques for platelet counting of dogs attended at Veterinary Hospital \\ of Universidade Estadual de Londrina (V.H.-UEL)
}

\author{
Patrícia Fernandes Nunes da Silva ${ }^{1 *}$; Mara Regina Stipp Balarin ${ }^{2} ;$ Helem Paula \\ Maruchi $^{3}$; Karina Keller Marques da Costa Flaiban ${ }^{4}$; Ludmila Rodrigues Moroz ${ }^{5}$
}

\section{Resumo}

Foram realizadas contagens plaquetárias em 30 cães para investigação diagnóstica. Para cada cão, foram obtidas contagens de plaquetas utilizando-se hemocitômetro manual e comparadas com contagens realizadas a partir da estimativa no esfregaço sangüíneo e pelo contador eletrônico por impedância MS4 ${ }^{\circledR}$. Nenhum dos dois métodos apresentou alta correlação com a contagem feita em câmara de Neubauer; o analisador por impedância apresentou a maior correlação.

Palavras-chave: Contagem de plaquetas. Cães. Hemocitômetro.

\begin{abstract}
Platelets counts were performed in 30 dogs for diagnostic investigation. For each dog, platelets counts were obtained using a manual haemocytometer and compared with counts obtained by estimation from a blood smear and a MS4 ${ }^{\circledR}$ analyzer. None of the two methods gave high correlation with the count obtained by a Neubauer chamber. The analyzer gave the highest correlation.
\end{abstract}

Key words: Platelet counting. Dogs. Haemocytometer.

\footnotetext{
1 Médica Veterinária formada pela Universidade Estadual de Londrina em 2005, atualmente é residente da área de Patologia Animal, sub-área em Patologia Clínica Veterinária da UEL, Departamento de Medicina Veterinária Preventiva - CCA/UEL. Email: patriciafnuness@yahoo.com.br.

${ }^{2}$ Médica Veterinária, Professora Associada, Departamento de Medicina Veterinária Preventiva - CCA/ Universidade Estadual de Londrina - PR.

3 Médica Veterinária, Autônoma / Maringá - PR

4 Médica Veterinária, Pós graduanda em Ciência Animal, Departamento de Medicina Veterinária Preventiva - CCA/ Universidade Estadual de Londrina - PR

5 Médica Veterinária, Professora, Centro Integrado de Campo Mourão - PR

* Autor para correspondência
} 


\section{Introdução}

Muitos diagnósticos hematológicos e conclusões de algumas patologias ocorrem devido à contagem anormal de células sangüíneas (TVEDTEN, 1993). A contagem de plaquetas com exatidão vem assumindo crescente importância em pacientes críticos, particularmente naqueles que apresentam trombocitopenia (OLIVEIRA et al., 2003). Em todos os animais com suspeita de anormalidades hemostáticas a contagem de plaquetas deve ser realizada rotineiramente (HACKNER, 1995).

O método pela diluição do sangue total e lise dos eritrócitos em hemocitômetro (câmara de Neubauer) é considerado o método oficial de referência para a contagem plaquetária pelo Comitê Internacional para Padronização em Hematologia (ICSH) (TASKER; CRIPPS; MACKIN, 2001). Os métodos manuais de contagem plaquetária, como o hemocitômetro e o esfregaço sangüíneo mantêm importante papel nos laboratórios clínicos veterinários que utilizam analisadores hematológicos avançados, já que a contagem realizada pelos métodos automáticos se mostra inexata em pacientes trombocitopênicos (TVEDTEN, 1993).

O uso da câmara de Neubauer é recomendado na presença de agregados plaquetários, em casos de suspeita de erro ao exame do esfregaço sangüíneo e em animais que apresentam trombocitopenia severa, visto que essa condição é uma causa de hemorragia espontânea e caracteriza um importante diagnóstico de inflamações ocultas e neoplasia em cães (OLSEN et al., 2004).

A avaliação microscópica do esfregaço sangüíneo é uma rápida estimativa do número de plaquetas obtida pela média contada por campo visualizado em aumento de 1000 x (TASKER; CRIPPS; MACKIN, 2001), sendo esse método utilizado como referência para a calibração dos contadores automáticos e parte essencial para a avaliação hematológica em animais doentes (SEGAL et al., 2005). Os resultados dos contadores automáticos podem ser suficientes sem a análise do esfregaço sangüíneo para a triagem de animais saudáveis. O esfregaço deve ser avaliado para a realização de uma estimativa do número de plaquetas (inclusive em animais trombocitopênicos), quando há tendência dessas a se agregarem e para a observação de sua morfologia, pois isso pode influenciar significantemente a contagem em métodos automáticos (TVEDTEN, 1993). Entretanto, a preparação de um esfregaço com qualidade para a contagem de plaquetas, bem fixado e com as células bem distribuídas, requer alguma prática, e os clínicos às vezes não se sentem seguros ao utilizar essa técnica (TASKER; CRIPPS; MACKIN, 2001).

Os métodos manuais, entretanto, despendem muito tempo e dependem da experiência do operador (OLSEN et al., 2004) e seu uso é economicamente justificável para um pequeno número de amostras (THIBODEAUX et al., 1989).

Atualmente, há um aumento do uso de analisadores hematológicos automáticos introduzindo uma variedade de novas tecnologias com interesse em se reduzir o trabalho manual e aumentar a exatidão da contagem. $\mathrm{O}$ maior avanço na contagem plaquetária tem se desenvolvido utilizando a citometria de fluxo (SEGAL et al., 2005). Os contadores de células sangüíneas modernos apresentam alto nível de precisão, mas a variação entre os instrumentos deve ser considerada (LEWIS; ROWAN; KUBOTA, 1990). Apesar dos analisadores automáticos sustentarem uma contagem completa sangüínea, eles são conhecidos por serem imprecisos na contagem de plaquetas em pacientes com trombocitopenia severa (LEWIS; ROWAN; KUBOTA, 1990). Os tipos de contadores hematológicos automáticos mais utilizados na rotina veterinária são contadores eletrônicos por impedância e contadores a laser.

Os analisadores por impedância diluem as células do sangue em um condutor elétrico, deixando as células passarem por uma pequena abertura entre dois eletrodos. A mudança na impedância elétrica gerada é proporcional ao tamanho da célula à medida que essa passa pela abertura (OLSEN et al., 2004).

A contagem de plaquetas realizada por sistemas 
a laser (citometria de fluxo) mede o desvio da luz em dois ângulos à medida que as células passam pelo raio (TVEDTEN, 1993). Eles são capazes de obter contagens plaquetárias precisas utilizando anticorpos plaquetários específicos e indicadores de cor.

As variações dos custos e o critério para a utilização de uma técnica para a contagem de plaquetas em cães devem ser considerados juntamente com as limitações de cada metodologia empregada. O presente artigo visa verificar a correlação de dois métodos utilizados na rotina laboratorial para a determinação do número de plaquetas em cães (estimativa do número de plaquetas em esfregaço e contagem plaquetária em contador automático) comparado ao método oficial de contagem de plaquetas (hemocitômetro).

\section{Material e Métodos}

Os animais selecionados foram cães triados para a Clínica Médica ou Cirúrgica de Animais de Companhia no atendimento de rotina do Hospital Veterinário da Universidade Estadual de Londrina. A escolha foi feita independentemente da idade e raça do animal. O estudo realizou-se a partir do encaminhamento de 30 amostras de sangue, cada uma contendo de 3 a $5 \mathrm{~mL}$, colhidas e armazenadas em tubos de vidro contendo anticoagulante etilenodiamino-tetraacetato (EDTA) na concentração 5\%. As veias de escolha foram a jugular e cefálica que são as vias mais comumente acessadas no H.V.UEL. As amostras de sangue foram analisadas no Laboratório Clínico do Departamento de Medicina Veterinária Preventiva da UEL, efetuando-se a contagem de plaquetas por três diferentes métodos: contagem em câmara de Neubauer, estimativa do número em esfregaço sangüíneo e contagem realizada em contador eletrônico por impedância. Os valores de referência para a contagem de plaquetas utilizados no estudo estão entre 200.000 e 500.000 células/ $\mu \mathrm{L}$, segundo Jain (1986).

\section{Contagem de plaquetas em câmara de Neubauer}

A contagem de plaquetas em câmara de Neubauer foi realizada segundo Jain (1986). Utilizou-se como diluidor o oxalato de amônio a $1 \%$ para a lise das hemácias. Em $10 \mu \mathrm{L}$ de sangue recém coletado foi acrescentado $2 \mathrm{~mL}$ do diluidor de Brecher e homogeneizado por 5 minutos. Os dois compartimentos da câmara foram preenchidos. O hemocitômetro foi deixado em ambiente úmido durante 20 minutos, colocando o mesmo em uma placa de Petri contendo papel absorvente umedecido, para que as plaquetas se sedimentassem no fundo da câmara. A iluminação do microscópio óptico foi reduzida para um maior contraste e visualização das plaquetas. Cinco campos de cada compartimento foram contados, semelhantemente ao método utilizado para contagem de hemácias, sendo o resultado multiplicado por 2.500 , obtendo-se o número de plaquetas $/ \mu \mathrm{L}$.

\section{Estimativa de plaquetas no esfregaço}

O esfregaço sangüíneo foi confeccionado numa lâmina de vidro logo após a coleta da amostra, corando-o com Panótico rápido (Diff-Quick) e visualizado em objetiva de imersão com aumento de $1000 \mathrm{x}$. A escolha do campo foi baseada numa igual distribuição das plaquetas. A média do número de plaquetas foi determinada contando-se cinco campos, multiplicando o resultado pelo fator 15.000, obtendo o número de plaquetas/ $\mu \mathrm{L}$; esta metodologia é utilizada para a contagem de plaquetas no Laboratório Clínico do H.V.-UEL. Partículas não-celulares e debris foram facilmente diferenciados das plaquetas.

\section{Contagem de plaquetas no contador eletrônico}

O contador automático utilizado foi o MS4 ${ }^{\circledR}$ o qual se baseia no sistema de contagem elétrica (também conhecido por impedância) com princípio de que as células são diluídas num fluido isotônico com alta condutividade elétrica. Há dois eletrodos separados 
por um tubo de vidro que tem uma pequena abertura. A corrente elétrica é conduzida pelo fluido através dos eletrodos. As células sangüíneas, no entanto, não são boas condutoras elétricas e quando passam pela abertura, impedem transitoriamente que a corrente elétrica flua numa zona sensível que detecta a voltagem, possibilitando a sua contagem. A duração e amplitude do impulso são proporcionais ao volume da célula. Para a realização de uma análise é necessário um cartão MS (que é enviado junto com o kit de reagentes) habilitando assim o controle e a validade dos reagentes. O MS4 ${ }^{\circledR}$ efetua dois tipos de aplicações, uma humana e outra veterinária, sendo específico para cada aplicação.

Os dados da contagem plaquetária obtidos pelos métodos do hemocitômetro, pela estimativa em esfregaço sangüíneo e pelo contador automático por impedância foram registrados em Excel e no programa Saeg para a análise estatística descritiva e determinação do coeficiente de correlação. A contagem manual em câmara de Neubauer foi escolhida como a 'prova-ouro' (JAIN, 1986) e comparada com os outros dois métodos de contagem de plaquetas.

\section{Resultados}

Os resultados encontrados no estudo estão ilustrados na Tabela 1. O método do hemocitômetro revelou número de plaquetas dentro da normalidade em 10 amostras e contagem abaixo, em 19 amostras. Apenas um animal apresentou contagem acima de 500.000 plaquetas $/ \mu \mathrm{L}$. A estimativa do número de plaquetas realizada através de esfregaço sangüíneo mostrou que em 14 amostras a contagem estava abaixo do normal e em três animais o número estava acima do valor. A contagem realizada pelo contador automático revelou contagem abaixo do normal em 11 animais e contagem acima em 4 amostras.

Os resultados de média e desvio padrão para contagem de plaquetas para os métodos estão ilustrados na Tabela 2. O método automático revelou a maior média de contagem de plaquetas (aproximadamente 280.000 células/ $\mu \mathrm{L}$ ). A correlação entre os métodos e a contagem manual em câmara de Neubauer é mostrada na Tabela 3.

Todos os métodos tiveram valores de correlação intermediários, com os coeficientes abaixo de $\mathrm{r}=0.6$. A contagem de plaquetas realizada pelo contador por impedância teve a maior correlação $(r=0.5991)$. A estimativa do número de plaquetas em esfregaço sanguiíneo teve uma correlação próxima ao da contagem automática, mas com valor menor ( $\mathrm{r}=$ $0.5688)$.

\section{Discussão}

A maior correlação de contagem de plaquetas com o método do hemocitômetro foi obtida pelo método automático. Em várias espécies animais o método eletrônico (ou por impedância) tem sido muito utilizado e aceito como um método padrão para uma contagem exata do número de plaquetas, apesar de suas falhas (KNOLL; ROWAN, 1996). Há uma tendência de todos os analisadores automáticos em superestimar a contagem de plaquetas, resultando em pouca sensibilidade a níveis abaixo do limite, mas há boa especificidade acima do limite de referência (SEGAL et al., 2005). A maior média do número de plaquetas obtida neste estudo dentre as três metodologias de contagem realizadas foi alcançada pelo analisador por impedância (280.000 células/ $\mu \mathrm{L})$, conforme os dados de outros relatos. 
Tabela 1. Contagem de plaquetas $(/ \mu \mathrm{L})$ em 30 cães, obtidos pelos métodos de contagem manual (câmara e esfregaço) e contagem automática.

\begin{tabular}{lccc}
\hline & Contador eletrônico & Esfregaço & Câmara \\
\hline $\mathbf{1}$ & 510.000 & 213.000 & 292.000 \\
$\mathbf{2}$ & 248.000 & 162.000 & 187.000 \\
$\mathbf{3}$ & 65.000 & 15.000 & 37.500 \\
$\mathbf{4}$ & 428.000 & 480.000 & 542.000 \\
$\mathbf{5}$ & 55.000 & 15.000 & 55.000 \\
$\mathbf{6}$ & 49.000 & 75.000 & 82.500 \\
$\mathbf{7}$ & 300.000 & 126.000 & 167.500 \\
$\mathbf{8}$ & 125.000 & 330.000 & 155.000 \\
$\mathbf{9}$ & 59.000 & 162.000 & 110.000 \\
$\mathbf{1 0}$ & 220.000 & 222.000 & 120.000 \\
$\mathbf{1 1}$ & 133.000 & 87.000 & 112.500 \\
$\mathbf{1 2}$ & 336.000 & 400.000 & 175.000 \\
$\mathbf{1 3}$ & 391.000 & 348.000 & 270.000 \\
$\mathbf{1 4}$ & 295.000 & 165.000 & 155.000 \\
$\mathbf{1 5}$ & 422.000 & 474.000 & 255.000 \\
$\mathbf{1 6}$ & 18.000 & 90.000 & 70.000 \\
$\mathbf{1 7}$ & 221.000 & 222.000 & 322.000 \\
$\mathbf{1 8}$ & 79.000 & 78.000 & 55.000 \\
$\mathbf{1 9}$ & 142.000 & 153.000 & 222.500 \\
$\mathbf{2 0}$ & 119.000 & 144.000 & 225.000 \\
$\mathbf{2 1}$ & 56.000 & 84.000 & 82.500 \\
$\mathbf{2 2}$ & 382.000 & 265.000 & 87.500 \\
$\mathbf{2 3}$ & 252.000 & 195.000 & 60.000 \\
$\mathbf{2 4}$ & 604.000 & 447.000 & 122.500 \\
$\mathbf{2 5}$ & 439.000 & 750.000 & 222.500 \\
$\mathbf{2 6}$ & 280.000 & 320.000 & 160.000 \\
$\mathbf{2 7}$ & 550.000 & 230.000 \\
$\mathbf{2 8}$ & 428.000 & 263.000 & 425.000 \\
$\mathbf{2 9}$ & 401.000 & 336.000 & 190.000 \\
$\mathbf{3 0}$ & 604.000 & 618.000 & 435.000 \\
\hline & 732.000 & &
\end{tabular}

Tabela 2. Média e Desvio Padrão da contagem de plaquetas em cães, obtidos pelos métodos de contagem manual (hemocitômetro e esfregaço) e contagem automática.

\begin{tabular}{ccc}
\hline & Média & $\begin{array}{c}\text { Desvio } \\
\text { Padrão }\end{array}$ \\
\cline { 2 - 3 } $\begin{array}{c}\text { Contagem manual no } \\
\text { hemocitômetro }\end{array}$ & 187.516 & 122.183 \\
Contagem no esfregaço sangüíneo & & \\
Contador automático MS4 & 259.633 & 183.903 \\
\hline
\end{tabular}

Tabela 3. Coeficiente de correlação ( $r$ ) da contagem manual de plaquetas em cães feita no hemocitômetro e os métodos de contagem em analisador e esfregaço.

\begin{tabular}{ccc}
\hline & $\begin{array}{c}\text { Analisador } \\
\text { MS4 }\end{array}$ & $\begin{array}{c}\text { Esfregaço } \\
\text { Sangüíneo }\end{array}$ \\
\cline { 2 - 3 } $\begin{array}{c}\text { Contagem manual no } \\
\text { hemocitômetro }\end{array}$ & 0.60 & 0.57 \\
$P$ & $<0.01$ & $<0.01$ \\
\hline
\end{tabular}


Sabe-se que os contadores por impedância têm inabilidade em diferenciar os eritrócitos e plaquetas, porque essas células possuem tamanhos semelhantes e isso pode resultar em uma contagem inexata de plaquetas (KNOLL; ROWELL, 1996). Sua precisão é limitada a baixos níveis de plaquetas, com um aumento na variabilidade da contagem pois há falha deste método em diferenciar as plaquetas de fragmentos celulares e debris (OLSEN et al., 2004). Isto pode resultar numa contagem plaquetária elevada ou, como no caso de plaquetas de maior tamanho, elas não serão diferenciadas das hemácias e a consequiência será uma falsa trombocitopenia. A contagem celular realizada através do analisador eletrônico revelou apenas 11 animais trombocitopênicos, enquanto que os outros dois métodos obtiveram um maior número de animais com contagem plaquetária abaixo dos valores de referência (14 e 19 animais trombocitopênicos pelo método do esfregaço e o oficial, respectivamente), revelando certa ineficácia para a contagem plaquetária na metodologia automática.

Por isso, a contagem de plaquetas através de métodos manuais é recomendável para cães que apresentam trombocitopenia. O maior número de cães trombocitopênicos foi obtido pelo método da câmara de Neubauer, totalizando 19 animais. Isso explica a média mais baixa do número de plaquetas verificada no método oficial (aproximadamente 188.000 células/ $\mu \mathrm{L})$. O método padrão utilizado para este estudo foi o do hemocitômetro porque mesmo com o uso da tecnologia, não se pode competir com o critério de discernimento humano (SUTOR et al., 2001).

\section{Conclusão}

O uso do método automático por impedância $\left(\mathrm{MS}^{\circledR}{ }^{\circledR}\right.$ na rotina laboratorial pode ser considerado confiável, já que se correlaciona com o método oficial em $60 \%$ das vezes em que se realizam contagens de plaquetas de cães, além de amenizar o tempo despendido pelo operador.

\section{Referências}

HACKNER, S. G. Aprroach to the diagnosis of bleeding disorders. The Compendium on Continuing Education for the Practicing Veterinarian, Princeton, v.17, n.3, p.331350,1995 .

JAIN, N. C. The platelets: structural, biochemical and functional aspects. In: Schalm's veterinary hematology. 4.ed. Philadelphia: Lea \& Febiger, 1986. p.1-4.

KNOLL, J. S.; ROWELL, S. Clinical hematology in clinic analysis, quality control, reference values and system selection. Veterinary Clinics of North America: Small Animal Practice, Philadelphia, v.26, n.5, p.981-1002, 1996.

LEWIS, S. M.; ROWAN, R. M.; KUBOTA, F. Evaluation of prototype for a reference platelet counter. Journal of Clinical Pathology, London, v.43, n.11, p.932-936, 1990.

OLIVEIRA, R. A. G.; TAKADACHI, M. M.; NONOYAMA, K.; BARRETTO, O. C. O. Is automated platelet counting still a problem in thrombocytopenic blood? São Paulo Medical Journal, São Paulo, v.121, n.1, 2003.

OLSEN, L. H.; KRISTENSEN, A. T.; QVORTRUP, K.; PEDERSEN, H. D. Comparison of manual and automated methods for determining platelet counts in dogs with macrothrombocitopenia. Journal of Veterinary Diagnostic Investigation, Columbia, v.16, n.2, p.167-170, 2004.

SEGAL, H. C.; BRIGGS, C.; KUNKA, S.; CASBARD, A.; HARRISON, P.; MACHIN, S. J.; MURPHY, M. F. Accuracy of platelet counting haematology analysers in severe thrombocitopenia and potential impact on platelet transfusion. British Journal of Haematology, Oxford, v.128, n.4, p.520-525, 2005.

SUTOR, A. H.; GROHMANN, A.; KAUFMEHL, K.; WÜNDISCH, T. Problems with platelet counting in thrombocitopenia. a rapid manual method to measure low platelets Counts. Seminars in Thrombosis and Hemostasis, New York, v.27, n.3, p.237-243, 2001.

TASKER, S.; CRIPPS, P. J.; MACKIN, A. J. Evaluation of methods of platelet counting in the cat. Journal of Small Animal Practice, Oxford, v.42, n.7, p.326-332, 2001.

THIBODEAUX, J. K.; ROUSSEL, J. D.; ADKINSON, R. W.; GOODEAUX, L. L. An efficient procedure for manual platelet counting. Veterinary Record, London, v.125, n.16, p.417-419, 1989.

TVEDTEN, H. Advanced hematology analyzers interpretation of results Veterinary Clinical Pathology, Santa Barbara, v.22, n.3, p.72-80, 1993. 